Reductions of integrable equations: dihedral group

Lombardo, S. and Mikhailov, A.V.

2004

MIMS EPrint: 2007.151

Manchester Institute for Mathematical Sciences

School of Mathematics

The University of Manchester

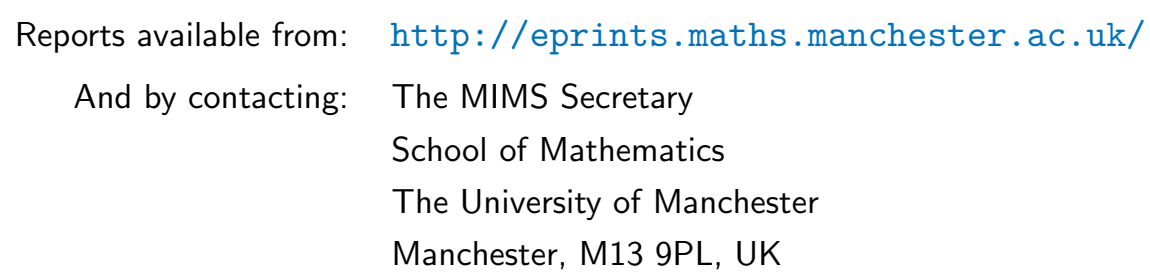

ISSN 1749-9097 


\title{
Reductions of integrable equations: dihedral group
}

\author{
S Lombardo ${ }^{1}$ and A V Mikhailov ${ }^{1,2}$ \\ ${ }^{1}$ Department of Applied Mathematics, University of Leeds, Leeds LS2 9JT, UK \\ ${ }^{2}$ L D Landau Institute for Theoretical Physics, Chernogolovka, Russia (on leave) \\ E-mail: sara@maths.leeds.ac.uk and sashamik@maths.leeds.ac.uk
}

Received 1 April 2004

Published 21 July 2004

Online at stacks.iop.org/JPhysA/37/7727

doi:10.1088/0305-4470/37/31/006

\begin{abstract}
We discuss the algebraic and analytic structure of rational Lax operators. With algebraic reductions of Lax equations we associate a reduction group — a group of automorphisms of the corresponding infinite-dimensional Lie algebra. We present a complete study of dihedral reductions for $\operatorname{sl}(2, \mathbb{C})$ Lax operators with simple poles and corresponding integrable equations. In the last section we give three examples of dihedral reductions for $\operatorname{sl}(N, \mathbb{C})$ Lax operators.
\end{abstract}

PACS numbers: $02.30 . \mathrm{Ik}, 02.30 . J r, 02.30 . \mathrm{Zz}$

\section{Introduction}

The inverse spectral transform method (IST) for integrating nonlinear partial differential equations (PDEs) is based on the Lax representation (see for example [1-3]). In this setting, the nonlinear equation is equivalent to the compatibility condition of a pair of linear equations, called a Lax pair. In general, given an integrable nonlinear equation, there is no algorithmic way to find its Lax representation. Some progress in the problem of finding a Lax representation for a given nonlinear equation has been made by the Wahlquist and Estabrook method of pseudo-potentials and prolongation structures [4-6]. In some cases the Painlevé approach provides both integrability conditions and Lax representation (see for example [7]).

A different approach to this problem consists in starting from a quite general Lax representation which yields to a rather big (but integrable) system of equations. In general, such a system has too many degrees of freedom and is very complicated. However, it can contain smaller subsystems, relevant for applications: many famous integrable equations (such as the sine-Gordon equation and the Tzetseica equation) are indeed the result of reductions of more general integrable systems. This observation suggests the study and classification of possible Lax representations and their reductions.

The first attempt to study algebraic reductions of Lax representations has been made in [8-10] and later in [11]. The principal observation in the papers [8,9] was that reductions 
of integrable equations can be associated with a discrete symmetry group (called a reduction group) of the corresponding linear problems. The simplest example of such a symmetry is the conjugation for self-adjoint operators.

In this paper, which is meant to be the first of a series, we report new developments of this approach. The paper is organized as follows. In section 2 we revisit the concept of Lax representation and its reductions. We define a quite general family of Lax operators with rational dependence on the spectral parameter $\lambda$, on a simple Lie algebra $\mathfrak{g}$. In section 3 we study reductions of integrable equations corresponding to rational Lax pairs, with simple poles in $\lambda$ and $\mathfrak{g}=\operatorname{sl}(2, \mathbb{C})$. Section 4 concentrates on the study of possible reductions of a fairly general Lax pair whose underlying algebra is $\mathfrak{g}=s l(N, \mathbb{C})$. In both sections, reductions are obtained using realizations of the reduction group isomorphic to the dihedral group $\mathbb{D}_{N}$. Some of the systems of equations obtained in these sections are new, to the best of our knowledge. Section 5 is devoted to conclusions and remarks.

\section{Lax representation of integrable systems}

Let us start this preliminary section reviewing the concept of Lax representation of an integrable system. Here and afterwards subscripts mean partial differentiation.

\subsection{Example of a rational matrix Lax pair and gauge transformations}

Let us consider two linear equations

$$
L \psi=\psi_{x}-Q(x, t) \psi=0, \quad M \psi=\psi_{t}-P(x, t) \psi=0,
$$

where $\psi, Q$ and $P$ are $N \times N$ matrix functions of $x$ and $t$. This is an over-determined system of equations and its compatibility condition

$$
Q_{t}-P_{x}+[Q, P]=0
$$

is equivalent to a system of nonlinear partial differential equations (PDEs). Its general solution is given by

$$
Q=g_{x} g^{-1}, \quad P=g_{t} g^{-1},
$$

where $g=g(x, t)$ is any nonsingular matrix function of $x$ and $t$. The same problem becomes nontrivial if one assumes that the matrices $Q$ and $P$ depend also on an auxiliary parameter $\lambda$, called spectral parameter, and are polynomial or rational functions of this parameter. As a consequence, equation (2) has to be satisfied for all values of $\lambda$ and this requirement leads to a nontrivial system of integrable, partial differential equations. In the latter case, one has to solve a certain matrix Riemann-Hilbert problem to find a suitable matrix function $g(x, t, \lambda)$, which would provide the solution of the system (see for example [1, 12, 13]). In order to receive a nontrivial set of nonlinear equations integrable by the IST method an essential dependence of $Q$ and $P$ in (2) on the spectral parameter $\lambda$ is required, where essential dependence on $\lambda$ means that it cannot be eliminated by a $\lambda$ dependent gauge transformation (see below).

As an example, let us consider the case of $Q$ and $P$ given by

$$
Q=Q_{0}+\sum_{n=1}^{N_{1}} \frac{Q_{n}}{\lambda-a_{n}}, \quad P=P_{0}+\sum_{n=1}^{N_{2}} \frac{P_{n}}{\lambda-b_{n}},
$$

where it is assumed that the sets of complex constants $\left\{a_{1}, \ldots, a_{N_{1}} \mid a_{n} \in \mathbb{C}\right\}$ and $\left\{b_{1}, \ldots, b_{N_{2}} \mid b_{n} \in \mathbb{C}\right\}$ have empty intersection and the matrix-coefficients $Q_{n}, P_{n}$ may depend on $x$ and $t$ only. Here and hereafter $\lambda \in \mathbb{C}$. Under these assumptions the compatibility condition (2) leads, for any $\lambda$, to a system of $N_{1}+N_{2}+1$ equations 


$$
\begin{aligned}
& Q_{0 t}-P_{0 x}+\left[Q_{0}, P_{0}\right]=0 \\
& Q_{n t}+\left[Q_{n}, P_{0}+\sum_{m=1}^{N_{2}} \frac{P_{m}}{a_{n}-b_{m}}\right]=0 \\
& P_{n x}+\left[P_{n}, Q_{0}-\sum_{m=1}^{N_{1}} \frac{Q_{m}}{a_{n}-b_{m}}\right]=0
\end{aligned}
$$

for $N_{1}+N_{2}+2$ functions, i.e. the system obtained is under-determined. Lax representations of this type and their reductions will be studied in section 3 .

The system of equations (4)-(6) is invariant with respect to the transformations

$$
Q \rightarrow g^{-1} Q g-g^{-1} g_{x}, \quad P \rightarrow g^{-1} P g-g^{-1} g_{t},
$$

where $g$ is any nonsingular matrix function of $x$ and $t$ (in principle it may depend on the spectral parameter $\lambda$, but in this section we shall assume $g=g(x, t))$. These transformations are called gauge transformations $[12,14]$. In terms of matrices $Q_{n}$ and $P_{n}$ the gauge transformation (7) takes the form

$$
\begin{array}{ll}
Q_{0} \rightarrow g^{-1} Q_{0} g-g^{-1} g_{x}, & P_{0} \rightarrow g^{-1} P_{0} g-g^{-1} g_{t}, \\
Q_{n} \rightarrow g^{-1} Q_{n} g, & P_{n} \rightarrow g^{-1} P_{n} g,
\end{array}
$$

where $n \geqslant 1$. There are several ways to fix the gauge. The natural or canonical choice [12] is to set $Q_{0}=P_{0}=0$. This is possible because the equations

$$
Q_{0} g-g_{x}=0, \quad P_{0} g-g_{t}=0
$$

are compatible and their compatibility condition coincides with (4). In this gauge, the system becomes a well determined system of $N_{1}+N_{2}$ matrix equations (5), (6) for $N_{1}+N_{2}$ matrix functions $Q_{n}, P_{n}, n \geqslant 1$. Another useful way to fix the gauge is to assume that the matrix $Q_{1}$ is in a canonical (for instance diagonal) form. If the eigenvalues of $Q_{1}$ are distinct, then the remaining gauge freedom, i.e. transformations (8) which do not change $Q_{1}$, consists of nonsingular diagonal matrices $g$. This remaining freedom can be used to make the matrix $Q_{0}$ off-diagonal. Such choice of the gauge is called the pole gauge [12] and it also provides a well determined system of equations.

The nature of the gauge transformations is obvious: the compatibility condition (2) is the commutativity condition of two linear differential operators

$$
L=\partial_{x}-Q, \quad M=\partial_{t}-P .
$$

If the operators $L$ and $M$ commute $([L, M]=0)$, then the transformed operators

$$
\hat{L}=g^{-1} L g, \quad \hat{M}=g^{-1} M g
$$

also commute. Transformations of the form

$$
\hat{L}=-h^{-1} L^{A} h, \quad \hat{M}=-h^{-1} M^{A} h,
$$

where $L^{A}=-\partial_{x}+Q^{\text {tr }}, M^{A}=-\partial_{x}+P^{\text {tr }}$ stand for formally adjoint operators and 'tr' stands for matrix transposition, are also gauge transformations. Gauge transformations form a group, the gauge group.

In the gauge transformations (9), (10) the invertible matrices $g, h$ may depend on $x, t, \lambda$ or even be differential or pseudo-differential matrix operators. The Miura and Bäcklund transformations can be viewed as special kind of gauge transformations. 


\subsection{Lax representations and Lie algebras}

Equations (4)-(6) are naturally defined on any Lie algebra $\mathfrak{g}$. Indeed, if $Q_{n}, P_{n} \in \mathfrak{g}$, then all commutators and derivatives also belong to $\mathfrak{g}$. Let $\mathfrak{g}$ be a finite-dimensional Lie algebra over $\mathbb{C}$. It is easy to see that only simple algebras are related to coupled nonlinear equations. Indeed, according to the Levi-Maltsev theorem [15], any finite dimensional Lie algebra $\mathfrak{g}$ over $\mathbb{C}$ can be decomposed as a direct sum

$$
\mathfrak{g}=\mathcal{R} \oplus \mathcal{S}
$$

where $\mathcal{R}$ is a solvable radical of $\mathfrak{g}$ and $\mathcal{S}$ is a semi-simple subalgebra. The semi-simple subalgebra $\mathcal{S}$, if nontrivial, is a sum of simple subalgebras

$$
\mathcal{S}=\oplus \mathcal{S}_{k}
$$

and the following commutation relations hold:

$$
[\mathcal{R}, \mathcal{R}] \subset \mathcal{R}, \quad\left[\mathcal{S}_{k}, \mathcal{R}\right] \subset \mathcal{R}, \quad\left[\mathcal{S}_{k} \mathcal{S}_{n}\right]=\delta_{k, n} \mathcal{S}_{k} .
$$

If we decompose $Q$ and $P$ in (2) according to the Levi-Maltsev decomposition theorem

$$
Q=Q_{\mathcal{R}}+\sum Q_{\mathcal{S}_{k}}, \quad P=P_{\mathcal{R}}+\sum P_{\mathcal{S}_{k}},
$$

we obviously receive

$$
\begin{aligned}
& \partial_{t} Q_{\mathcal{S}_{k}}-\partial_{x} P_{\mathcal{S}_{k}}+\left[Q_{\mathcal{S}_{k}}, P_{\mathcal{S}_{k}}\right]=0 \\
& \partial_{t} Q_{\mathcal{R}}-\partial_{x} P_{\mathcal{R}}+\left[Q_{\mathcal{R}}, P_{\mathcal{R}}\right]+\left[Q_{\mathcal{R}}, \sum P_{\mathcal{S}_{k}}\right]+\left[\sum Q_{\mathcal{S}_{k}}, P_{\mathcal{R}}\right]=0 .
\end{aligned}
$$

We see that equations (11), corresponding to the semi-simple part, can be treated separately from the rest of the system and there is no coupling between equations corresponding to different simple subalgebras. It is easy to show that, in the radical $\mathcal{R}$, one can choose a basis in which the system of equations (12) becomes 'triangular', i.e. it is an ordered chain of equations such that each equation in this chain is a linear equation with respect to its own dependent variable and with coefficients and an inhomogeneous part depending on variables of the preceding equations and variables corresponding to the semi-simple part. Therefore, the problem of integration of the whole system is reduced to the integration of a closed system of the nonlinear equations (11) and then to the integration of a chain of linear equations (12) with variable coefficients.

In this paper we focus our attention on the study of nonlinear equations and therefore we shall always assume that the underlying algebra $\mathfrak{g}$ is simple (or semi-simple).

\subsection{General rational Lax operators}

In this section we define a quite general family of Lax operators with rational dependence on the spectral parameter $\lambda$. Let $\mathfrak{g}$ be a finite-dimensional simple Lie algebra over $\mathbb{C}$ and let $\left\{\mathfrak{g}_{1}, \ldots, \mathfrak{g}_{N}\right\}$ be a basis of $\mathfrak{g}$

$$
\left[\mathfrak{g}_{i}, \mathfrak{g}_{j}\right]=\sum_{r=1}^{N} C_{i j}^{r} \mathfrak{g}_{r}, \quad C_{i j}^{r} \in \mathbb{C} .
$$

Let $X=\left\{x_{1}, x_{2}, \ldots\right\}$ be a set of independent variables (in the previous example (1) we used $x$ and $t$ variables). With every variable $x_{k}$ we associate a divisor of poles $\Gamma_{k}=m_{1} \cdot \gamma_{1}+\cdots+m_{n} \cdot \gamma_{n}$, i.e. a finite set of points $\hat{\Gamma}_{k}=\left\{\gamma_{1}, \ldots, \gamma_{n}\right\}$ on the Riemann sphere together with their multiplicities $m_{1}, \ldots, m_{n} \in \mathbb{N}$. Let $\mathcal{L}\left(\Gamma_{k}\right)$ denote a linear space of 
rational functions of the spectral parameter $\lambda$ such that the only singularities of these functions are poles at the points of the divisor with multiplicity equal or less than the multiplicity of the point. The dimension of this linear space $\operatorname{dim} \mathcal{L}\left(\Gamma_{k}\right)$ is $M_{k}=m_{1}+\cdots+m_{n}+1$. Let $e_{1}^{k}(\lambda), \ldots, e_{M_{k}}^{k}(\lambda)$ be a basis in $\mathcal{L}\left(\Gamma_{k}\right)$.

With $x_{k}, \Gamma_{k}$ and $\mathfrak{g}$ we associate the general Lax operator

$$
L_{k}=\frac{\mathrm{d}}{\mathrm{d} x_{k}}-\sum_{r=1}^{N} \sum_{\beta=1}^{M_{k}} \mathfrak{g}_{r} e_{\beta}^{k}(\lambda) u_{r \beta}^{k},
$$

where $u_{r \beta}^{k}$ are smooth functions of variables $X$ in a certain open domain, and if the set $X$ is infinite, we assume that every function depends on a finite number of variables only. A set of such functions is denoted by $\mathcal{F}$.

The Lax operator $L_{k}$ is parametrized by $N \times M_{k}$ functions $u_{r \beta}^{k}$. Any two Lax operators $L_{k}, L_{s}$ form a Lax pair. The commutativity condition $\left[L_{k}, L_{s}\right]=0$ is equivalent to an integrable (by the spectral transform method) system of partial differential equations for the functions $u_{r \beta}^{k}, u_{r^{\prime} \beta^{\prime}}^{s} \in \mathcal{F}$.

In order to write this system of PDEs in explicit form we introduce a basis $h_{\alpha}^{k s}(\lambda), \alpha=$ $1, \ldots, M_{k}+M_{s}-1$ in $\mathcal{L}\left(\Gamma_{k}+\Gamma_{s}\right)$ and expand elements $e_{\beta}^{k}(\lambda), e_{\beta^{\prime}}^{s}(\lambda) \in \mathcal{L}\left(\Gamma_{k}+\Gamma_{s}\right)$ and the products $e_{\beta}^{k}(\lambda) e_{\beta^{\prime}}^{s}(\lambda) \in \mathcal{L}\left(\Gamma_{k}+\Gamma_{s}\right)$ in this new basis

$$
\begin{array}{ll}
e_{\beta}^{k}(\lambda)=\sum_{\alpha=1}^{M_{k}+M_{s}-1} F_{\beta}^{k \alpha} h_{\alpha}^{k s}(\lambda), & e_{\beta}^{s}(\lambda)=\sum_{\alpha=1}^{M_{k}+M_{s}-1} F_{\beta}^{s \alpha} h_{\alpha}^{k s}(\lambda) \\
e_{\beta}^{k}(\lambda) e_{\beta^{\prime}}^{s}(\lambda)=\sum_{\alpha=1}^{M_{k}+M_{s}-1} H_{\beta \beta^{\prime}}^{k s \alpha} h_{\alpha}^{k s}(\lambda), & F_{\beta}^{k \alpha}, F_{\beta^{\prime}}^{s \alpha}, H_{\beta \beta^{\prime}}^{k s \alpha} \in \mathbb{C} .
\end{array}
$$

Thus we have

$$
\sum_{\beta=1}^{M_{k}} F_{\beta}^{k \alpha} \frac{\partial u_{r \beta}^{k}}{\partial x_{s}}-\sum_{\beta^{\prime}=1}^{M_{s}} F_{\beta^{\prime}}^{s \alpha} \frac{\partial u_{r \beta^{\prime}}^{s}}{\partial x_{k}}+\sum_{i, j=1}^{N} \sum_{\beta=1, \beta^{\prime}=1}^{M_{k}, M_{s}} C_{i j}^{r} H_{\beta \beta^{\prime}}^{k s \alpha} u_{i \beta}^{k} u_{i \beta^{\prime}}^{s}=0,
$$

where $r=1, \ldots, N$ and $\alpha=1, \ldots, M_{k}+M_{s}-1$.

Equation (16) is a system of nonlinear PDEs with constant coefficients. The system contains partial derivatives in variables $x_{k}$ and $x_{s}$ only, and if we assume that functions $u_{r \beta}^{k}, u_{r^{\prime} \beta^{\prime}}^{s}$ depend on other variables from the set $X$ we can treat these variables as parameters. The system obtained is canonical, it is uniquely defined by the choice of a simple Lie algebra $\mathfrak{g}$ and two divisors $\Gamma_{k}$ and $\Gamma_{s}$ (in some cases the divisors may coincide, cf the Lax pair for the $N$-wave equation [1]). Systems corresponding to a different choice of the basis of $\mathfrak{g}, \mathcal{L}\left(\Gamma_{k}\right), \mathcal{L}\left(\Gamma_{s}\right)$ and $\mathcal{L}\left(\Gamma_{k}+\Gamma_{s}\right)$ can be obtained from (16) by a linear invertible transformations of dependent variables.

The system of equations (16) is under-determined, it contains $N \times\left(M_{k}+M_{s}-1\right)$ equations on $N \times\left(M_{k}+M_{s}\right)$ dependent variables. Indeed, on the Riemann sphere, we have $\operatorname{dim} \mathcal{L}\left(\Gamma_{k}+\Gamma_{s}\right)=\operatorname{dim} \mathcal{L}\left(\Gamma_{k}\right)+\operatorname{dim} \mathcal{L}\left(\Gamma_{s}\right)-1$ (if we consider a similar setup on an algebraic curve with nonzero genus, then the genus would contribute in the accounting for the dimensions of the corresponding linear spaces of rational functions on the curve [16]). The difference between the number of dependent variables and the number of equations is equal to the dimension of the Lie algebra $\mathfrak{g}$. Fixing the gauge we finally obtain a well posed problem.

If we have a family of Lax operators $L_{1}, \ldots, L_{k}$ corresponding to divisors $\Gamma_{1}, \ldots, \Gamma_{k}$, then the conditions $\left[L_{i}, L_{j}\right]=0,1 \leqslant i<j \leqslant k$ yield an over-determined system of nonlinear partial differential equations. A general solution and explicit exact partial solutions of this system can be constructed by the spectral transform method. 


\subsection{The problem of reduction and the reduction group}

The integrable system (16) obtained in the previous section is very general. It looks too big and may not resemble useful equations but, up to the best of our knowledge, all known $(1+1)$ dimensional systems of integrable partial differential equations are subsystems (reductions) of (16). The problem to find and classify all subsystems of a general system is known as the reduction problem.

Restrictions to subalgebras $\hat{\mathfrak{g}} \subset \mathfrak{g}$ are obvious reductions of (16). For instance, in the system of equations (4)-(6) one can consider all matrices to be skew-symmetric and this is compatible with the dynamics since the commutator of skew-symmetric matrices is skew-symmetric. With this reduction we associate the automorphism $\phi: a \rightarrow-a^{\text {tr }}$ of the Lie algebra $\operatorname{sl}(N, \mathbb{C})$. The set of all elements of $\operatorname{sl}(N, \mathbb{C})$ which are invariant with respect to the automorphisms $\phi$ is obviously a subalgebra of skew-symmetric matrices $\operatorname{so}(N)=\{a \in \operatorname{sl}(N, \mathbb{C}) \mid a=\phi(a)\}$. A complete description of automorphisms of finitedimensional semi-simple Lie algebras is well known (see for example [15]). In the case of $\operatorname{sl}(N, \mathbb{C})$ automorphisms can be described as follows: for $\operatorname{sl}(2, \mathbb{C})$ all automorphisms are inner, i.e. they can be represented as $\phi: a \rightarrow G^{-1} a G$, where $G \in S L(2, \mathbb{C})$. For $\operatorname{sl}(N, \mathbb{C}), N>2$ the group of all automorphisms consists of inner $\phi: a \rightarrow G^{-1} a G$ and outer automorphisms $\psi: a \rightarrow-H^{-1} a^{\text {tr }} H, G, H \in S L(N, \mathbb{C})$.

Let $\mathcal{R}(\Gamma)$ be a ring of rational functions of variable $\lambda$ with poles at points of $\Gamma$ and regular elsewhere. Fractional-linear transformations of the complex plane $\lambda$, which map the divisor $\Gamma$ into itself, induce automorphisms of the ring $\mathcal{R}(\Gamma)$. We denote by $\operatorname{Aut} \mathcal{R}(\Gamma)$ the group of automorphisms of $\mathcal{R}(\Gamma)$. Any subgroup of $G_{R} \subset \operatorname{Aut} \mathfrak{g} \times \operatorname{Aut} \mathcal{R}(\Gamma)$ is a group of automorphisms of $\mathfrak{g}(\Gamma)=\mathfrak{g} \bigotimes_{\mathbb{C}} \mathcal{R}(\Gamma)$. The group $G_{R}$ is called reduction group. Elements of the reduction group can be viewed as simultaneous automorphisms, i.e. Lie algebra automorphisms and fractional-linear transformations of the spectral parameter $\lambda$. For example, in the case $\operatorname{sl}(N, \mathbb{C})$, the action of elements of the reduction group $G_{R}$ can be represented either as $a(\lambda) \rightarrow G^{-1} a\left(\sigma_{G}(\lambda)\right) G$ or as $a(\lambda) \rightarrow-H^{-1} a^{\operatorname{tr}}\left(\sigma_{H}(\lambda)\right) H$, where $G, H \in S L(N, \mathbb{C})$ and $\sigma_{G}(\lambda), \sigma_{H}(\lambda)$ are the corresponding fractional-linear transformations of the $\lambda$ plane.

The set $\mathfrak{g}_{G_{R}}(\Gamma)=\left\{a \in \mathfrak{g}(\Gamma) \mid a=\phi(a), \forall \phi \in G_{R}\right\}$ is a subalgebra of $\mathfrak{g}(\Gamma)$, which we shall call $G_{R}$-automorphic subalgebra. The restriction of the general Lax operator to the subalgebra $\mathfrak{g}_{G_{R}}(\Gamma)$ is a reduction, with reduction group $G_{R}$. Restrictions to invariant subalgebras are equivalent to require some symmetry conditions for the Lax operators. For example, in the case $\operatorname{sl}(N, \mathbb{C})$ they lead to symmetry conditions of the form

$$
L_{k}(\lambda)=G^{-1} L_{k}\left(\sigma_{G}(\lambda)\right) G, \quad L_{k}(\lambda)=-H^{-1} L_{k}^{A}\left(\sigma_{H}(\lambda)\right) H .
$$

Comparing with (9) and (10), we see that the operators are invariant with respect to simultaneous gauge transformations ${ }^{3}$ and fractional-linear transformations of the spectral parameter $\lambda$. The reduction group was originally introduced in $[8,9]$, our recent study of its algebraic structure and the corresponding automorphic Lie algebras will be published soon [17].

\section{3. $\mathbb{D}_{N}$-reductions of Lax operators with simple poles, $\mathfrak{g}=\operatorname{sl}(2, \mathbb{C})$}

In this section we study dihedral reductions of integrable equations corresponding to rational Lax pairs with simple poles in the spectral parameter $\lambda$ and $\mathfrak{g}=\operatorname{sl}(2, \mathbb{C})$.

\footnotetext{
3 As we have already mentioned at the end of section 2.1 there are further generalizations when $G$ and $H$ are coordinates and $\lambda$ dependent or even (pseudo) differential operators. We do not consider such generalized reductions in this paper.
} 
The dihedral group $\mathbb{D}_{N}$ is the group of rotations and reflections of the plane which preserve a regular polygon with $N$ vertices. It contains $N$ rotations, which form a normal subgroup isomorphic to $\mathbb{Z}_{N}$ and $N$ reflections; its order is $2 N$. If we denote by $s$ the rotation through an angle $2 \pi / N$ and if $r$ is any of the reflections, then each element of the group can be written uniquely either in the form $s^{k}$ or $r s^{k}, 0 \leqslant k \leqslant N-1$. In a more abstract way, the group $\mathbb{D}_{N}$ can be defined as the group generated by two elements, $s$ and $r$, satisfying the identities

$$
s^{N}=r^{2}=i d, \quad r s r=s^{-1} .
$$

In the case $N=2$ the group $\mathbb{D}_{2}$ is Abelian and isomorphic to $\mathbb{Z}_{2} \times \mathbb{Z}_{2}$.

On the complex plane of spectral parameter $\lambda$ the group $\mathbb{D}_{N}$ can be generated by two fractional-linear transformations

$$
\sigma_{s}: \lambda \rightarrow \omega \lambda, \quad \sigma_{r}: \lambda \rightarrow \lambda^{-1}, \quad \omega=\exp (2 \mathrm{i} \pi / N) .
$$

If a divisor $\Gamma$ is invariant with respect to transformations (19), it is a union of a finite number of orbits of the group $\mathbb{D}_{N}$. The orbit of the group $\mathbb{D}_{N}$ of a point $\gamma$ is defined as the set of points

$$
D_{N}(\gamma)=\left\{\sigma_{s}^{n}(\gamma), \sigma_{r} \sigma_{s}^{n}(\gamma) \mid n=1,2, \ldots N\right\} .
$$

When the point $\gamma$ is generic, i.e. $\gamma$ is not a fixed point of any (nontrivial) subgroup, the corresponding generic orbit has $2 N$ points

$\mathbb{D}_{N}(\gamma)=\left\{\gamma, \omega \gamma, \omega^{2} \gamma, \ldots, \omega^{N-1} \gamma, \gamma^{-1}, \omega \gamma^{-1}, \omega^{2} \gamma^{-1}, \ldots, \omega^{N-1} \gamma^{-1}\right\}$.

Fixed points of the group transformations (19) belong to degenerated orbits. There exists a degenerated orbit with two elements

$$
\mathbb{D}_{N}(0)=\{0, \infty\}
$$

and two degenerated orbits with $N$ elements. For odd $N$ they are

$\mathbb{D}_{N}(1)=\left\{\omega^{n} \mid n=1,2, \ldots, N\right\}, \quad \mathbb{D}_{N}(-1)=\left\{-\omega^{n} \mid n=1,2, \ldots, N\right\}$.

For even $N \mathbb{D}_{N}(1)=\mathbb{D}_{N}(-1)$ and the second orbit with $N$ points is

$$
\mathbb{D}_{N}\left(\omega^{1 / 2}\right)=\left\{\omega^{n+1 / 2} \mid n=1,2, \ldots, N\right\} .
$$

In the rest of this section we assume that $N$ is odd.

With every orbit we associate a linear space of rational functions with simple poles at the points of the orbit. Let $\gamma$ be a generic point and $\mathbb{D}_{N}(\gamma)$ the corresponding generic orbit. A natural way to construct a basis of rational functions in $\mathcal{L}\left(\mathbb{D}_{N}(\gamma)\right)$ is to start with $e_{0}=1, e_{1}=1 /(\lambda-\gamma)$ and apply all fractional-linear transformations of the group. As a result we receive a basis of $\mathcal{L}\left(\mathbb{D}_{N}(\gamma)\right)$

$$
e_{0}(\lambda)=1, \quad e_{k}(\lambda)=\frac{1}{\omega^{k-1} \lambda-\gamma}, \quad e_{N+k}(\lambda)=\frac{1}{\lambda-\omega^{k-1} \gamma}, \quad k=1,2, \ldots, N .
$$

This basis corresponds to the regular representation of the group $\mathbb{D}_{N}$. A regular representation can be decomposed into a direct sum of irreducible representations. Such a decomposition suggests another natural and useful basis of $\mathcal{L}\left(\mathbb{D}_{N}(\gamma)\right)$

$$
\begin{aligned}
& E_{0}^{\gamma}=1, \quad E_{1}^{\gamma}=\frac{\lambda^{N}+\gamma^{N}}{\lambda^{N}-\gamma^{N}}+\frac{1+\gamma^{N} \lambda^{N}}{1-\gamma^{N} \lambda^{N}}, \quad E_{2}^{\gamma}=\frac{\lambda^{N}+\gamma^{N}}{\lambda^{N}-\gamma^{N}}-\frac{1+\gamma^{N} \lambda^{N}}{1-\gamma^{N} \lambda^{N}}, \\
& E_{4 k-1}^{\gamma}=\frac{\lambda^{k}}{\lambda^{N}-\gamma^{N}}, \quad E_{4 k}^{\gamma}=\frac{\lambda^{N-k}}{1-\gamma^{N} \lambda^{N}}, \quad E_{4 k+1}^{\gamma}=\frac{\lambda^{k}}{1-\gamma^{N} \lambda^{N}}, \quad E_{4 k+2}^{\gamma}=\frac{\lambda^{N-k}}{\lambda^{N}-\gamma^{N}}, \\
& k=1, \ldots, \frac{N-1}{2} .
\end{aligned}
$$


The general Lax operator corresponding to a generic orbit $D_{N}(\gamma)$ is

$$
L_{\gamma}(\lambda)=\frac{\mathrm{d}}{\mathrm{d} x_{\gamma}}-\sum_{j=0}^{2 N+1} E_{j}^{\gamma}(\lambda) U_{j}^{\gamma},
$$

where $U_{j}^{\gamma}$ are $2 \times 2$ traceless matrix functions of independent variables (such as $x_{\gamma}$ ). Let $L_{\mu}(\lambda)$ be another Lax operator, corresponding to another generic orbit $D_{N}(\mu)$ and parametrized by matrix functions $U_{j}^{\mu}$. The condition $\left[L \gamma(\lambda), L_{\mu}(\lambda)\right]=0$ leads to a nonlinear system of $4 N+1$ matrix partial differential equations. We will reduce this huge system to a simple, well determined system of six scalar partial differential equations.

Let $a(\lambda) \in \operatorname{sl}(2, \mathbb{C}) \bigotimes_{\mathbb{C}} \mathcal{L}\left(\mathbb{D}_{N}(\gamma)\right)$, i.e.

$$
a(\lambda)=\sum_{j=0}^{2 N+1} E_{j}^{\gamma}(\lambda) a_{j}, \quad a_{j} \in \operatorname{sl}(2, \mathbb{C}),
$$

and consider the following linear transformations

$$
g_{s}: a(\lambda) \rightarrow S(h)^{-1} a\left(\sigma_{s}(\lambda)\right) S(h), \quad g_{r}: a(\lambda) \rightarrow R^{-1} a\left(\sigma_{r}(\lambda)\right) R,
$$

where $h$ is a fixed integer $1 \leqslant h \leqslant \frac{N-1}{2}$ that enumerates all different irreducible representations and

$$
S=S(h)=\left(\begin{array}{cc}
\omega^{-h} & 0 \\
0 & \omega^{+h}
\end{array}\right), \quad R=\left(\begin{array}{ll}
0 & 1 \\
1 & 0
\end{array}\right) .
$$

The transformations $g_{s}$ and $g_{r}$ generate a linear representation of $\mathbb{D}_{N}$ in the linear space $\operatorname{sl}(2, \mathbb{C}) \bigotimes_{\mathbb{C}} \mathcal{L}\left(\mathbb{D}_{N}(\gamma)\right)$. It is easy to check that (25) generate a subgroup of the group of automorphisms of Lie algebra $\operatorname{sl}(2, \mathbb{C}) \bigotimes_{\mathbb{C}} \mathcal{R}\left(\mathbb{D}_{N}(\gamma)\right)$. Let us consider invariant elements of $\operatorname{sl}(2, \mathbb{C}) \bigotimes_{\mathbb{C}} \mathcal{L}\left(\mathbb{D}_{N}(\gamma)\right)$, i.e. elements $a(\lambda)$ such that

$$
S(h)^{-1} a\left(\sigma_{s}(\lambda)\right) S(h)=a(\lambda), \quad R^{-1} a\left(\sigma_{r}(\lambda)\right) R=a(\lambda) .
$$

For every given $h$ the subspace of invariant elements is three dimensional; a basis of this space can be written as

$$
\mathcal{E}_{1}^{\gamma}=E_{2}^{\gamma}\left(\begin{array}{cc}
1 & 0 \\
0 & -1
\end{array}\right), \quad \mathcal{E}_{2}^{\gamma}=\left(\begin{array}{cc}
0 & E_{3}^{\gamma} \\
E_{4}^{\gamma} & 0
\end{array}\right), \quad \mathcal{E}_{3}^{\gamma}=\left(\begin{array}{cc}
0 & E_{5}^{\gamma} \\
E_{6}^{\gamma} & 0
\end{array}\right),
$$

where we fix $h=(N-1) / 2$ since the final result does not depend on the choice of the representation. Thus the reduced (invariant with respect to $\mathbb{D}_{N}$ reduction group (25)) Lax operator can be written as

$$
L_{\gamma}(\lambda)=\frac{\mathrm{d}}{\mathrm{d} x_{\gamma}}-q_{1} \mathcal{E}_{1}^{\gamma}-q_{2} \mathcal{E}_{2}^{\gamma}-q_{3} \mathcal{E}_{3}^{\gamma}
$$

where $q_{1}, q_{2}, q_{3}$ are scalar functions of independent variables. If

$$
L_{\mu}(\lambda)=\frac{\mathrm{d}}{\mathrm{d} x_{\mu}}-p_{1} \mathcal{E}_{1}^{\mu}-p_{2} \mathcal{E}_{2}^{\mu}-p_{3} \mathcal{E}_{3}^{\mu}
$$

is the second operator in the Lax pair, corresponding to another generic orbit $D_{N}(\mu)$, then the condition $\left[L_{\gamma}, L_{\mu}\right]=0$ leads to a system of integrable equations

$$
\begin{aligned}
& q_{1 t}+a\left(q_{2} p_{3}-p_{2} q_{3}\right) / 2+b\left(q_{2} p_{2}-q_{3} p_{3}\right) / 2=0 \\
& q_{2 t}-2 \beta p_{1} q_{2}+4 \gamma^{N}\left(a p_{2}+b p_{3}\right) q_{1}=0 \\
& q_{3 t}+2 \beta p_{1} q_{3}-4 \gamma^{N}\left(a p_{3}+b p_{2}\right) q_{1}=0 \\
& p_{1 x}+a\left(q_{2} p_{3}-p_{2} q_{3}\right) / 2+b\left(q_{2} p_{2}-q_{3} p_{3}\right) / 2=0 \\
& p_{2 x}+2 \alpha q_{1} p_{2}-4 \mu^{N}\left(a q_{2}-b q_{3}\right) p_{1}=0 \\
& p_{3 x}-2 \alpha q_{1} p_{3}+4 \mu^{N}\left(a q_{3}-b q_{2}\right) p_{1}=0
\end{aligned}
$$


where $x=x_{\gamma}, t=x_{\mu}$ and $a, b, \alpha, \beta$ are complex constants

$$
\begin{aligned}
& a=\left(\gamma^{N}-\mu^{N}\right)^{-1}, \quad b=\left(1-\gamma^{N} \mu^{N}\right)^{-1}, \\
& \alpha=\frac{\gamma^{N}+\mu^{N}}{\gamma^{N}-\mu^{N}}+\frac{1+\gamma^{N} \mu^{N}}{1-\gamma^{N} \mu^{N}}, \quad \beta=\frac{\gamma^{N}+\mu^{N}}{\gamma^{N}-\mu^{N}}-\frac{1+\gamma^{N} \mu^{N}}{1-\gamma^{N} \mu^{N}} .
\end{aligned}
$$

Clearly equations (29) can be rescaled and written in a different form, however they depend on parameters $\gamma^{N}$ and $\mu^{N}$, that cannot be removed by simple rescaling. What is surprising is that the resulting system does not depend on the choice of the representation. It does not depend on $N$ either! Indeed, equations corresponding to $N, \gamma, \mu$ coincide with equations corresponding to $N_{1}, \gamma_{1}, \mu_{1}$, provided the conditions $\gamma^{N}=\gamma_{1}^{N_{1}}$ and $\mu^{N}=\mu_{1}^{N_{1}}$ are satisfied. For the case of even $N$ we would receive equivalent equations, reflecting the fact that the corresponding automorphic Lie algebras are isomorphic [17].

For the orbits $\mathbb{D}_{N}( \pm 1)$ the bases of $\mathcal{L}\left(\mathbb{D}_{N}( \pm 1)\right)$ in which the representation are decomposed in a direct sum of irreducible ones can be written as

$$
\begin{aligned}
& F_{0}^{ \pm}(\lambda)=1, \quad F_{1}^{ \pm}(\lambda)=\frac{\lambda^{N} \pm 1}{\lambda^{N} \mp 1}, \quad F_{2 k}^{ \pm}(\lambda)=\frac{\lambda^{k}}{\lambda^{N} \mp 1}, \\
& F_{2 k+1}^{ \pm}(\lambda)=\frac{\lambda^{N-k}}{1 \mp \lambda^{N}}, \quad k=1, \ldots, \frac{N-1}{2} .
\end{aligned}
$$

The corresponding $\mathbb{D}_{N}$ invariant Lax operators are of the form

$$
L_{ \pm}(\lambda)=\frac{\mathrm{d}}{\mathrm{d} x_{ \pm}}-\frac{1}{2} u_{1}^{ \pm} F_{1}^{ \pm}(\lambda)\left(\begin{array}{cc}
1 & 0 \\
0 & -1
\end{array}\right)-u_{2}^{ \pm}\left(\begin{array}{cc}
0 & F_{2}^{ \pm}(\lambda) \\
F_{3}^{ \pm}(\lambda) & 0
\end{array}\right),
$$

where again we assume $h=(N-1) / 2$. The condition $\left[L_{+}, L_{-}\right]=0$ leads in this case to a rather simple system of equations

$u_{2 x_{-}}^{+}=u_{1}^{+} u_{2}^{-}, \quad u_{2 x_{+}}^{-}=u_{1}^{-} u_{2}^{+}, \quad u_{1 x_{-}}^{+}=u_{2}^{+} u_{2}^{-}, \quad u_{1 x_{+}}^{-}=u_{2}^{+} u_{2}^{-}$.

It follows from (30) that

$$
u_{2}^{+} u_{2 x_{-}}^{+}=u_{1}^{+} u_{1 x_{-}}^{+}, \quad u_{2}^{-} u_{2 x_{+}}^{-}=u_{1}^{-} u_{1 x_{+}}^{-},
$$

therefore we can partially integrate the equations and introduce the variables $\phi$ and $\theta$

$u_{1}^{+}=f\left(x_{+}\right) \cosh \phi, \quad u_{2}^{+}=f\left(x_{+}\right) \sinh \phi, \quad u_{1}^{-}=g\left(x_{-}\right) \cosh \theta, \quad u_{2}^{-}=g\left(x_{-}\right) \sinh \theta$, where $f\left(x_{+}\right)$and $g\left(x_{-}\right)$are two arbitrary functions (which can be set to be equal to 1 by a conformal transformation of the independent variables). In terms of $\phi$ and $\theta$ equations (30) are

$$
\phi_{x_{-}}=g\left(x_{-}\right) \sinh \theta, \quad \theta_{x_{+}}=f\left(x_{+}\right) \sinh \phi .
$$

They are nothing but a well known form of Bäcklund transformation for the sinh-Gordon equation. Indeed, equations (31) imply that

$$
\begin{aligned}
& (\theta+\phi)_{x_{+} x_{-}}=g\left(x_{-}\right) f\left(x_{+}\right) \sinh (\theta+\phi) \\
& (\theta-\phi)_{x_{+} x_{-}}=g\left(x_{-}\right) f\left(x_{+}\right) \sinh (\theta-\phi) .
\end{aligned}
$$

Let us take $L_{\gamma}$ and $L_{+}$as a Lax pair. The condition $\left[L_{\gamma}, L_{+}\right]=0$ is equivalent to the following system of equations

$$
\begin{aligned}
& u_{1 x_{\gamma}}^{+}=\frac{1}{\gamma^{N}-1}\left(q_{2}+q_{3}\right) u_{2}^{+} \\
& u_{2 x_{\gamma}}^{+}=-\frac{2}{\gamma^{N}-1}\left(q_{2}+q_{3}\right) u_{1}^{+}
\end{aligned}
$$




$$
\begin{aligned}
& q_{1 x_{+}}=\frac{1}{2\left(\gamma^{N}-1\right)}\left(q_{2}+q_{3}\right) u_{2}^{+} \\
& q_{2 x_{+}}=-\frac{4 \gamma^{N}}{\gamma^{N}-1} q_{1} u_{2}^{+}+\frac{2\left(\gamma^{N}+1\right)}{\gamma^{N}-1} q_{2} u_{1}^{+} \\
& q_{3 x_{+}}=-\frac{4 \gamma^{N}}{\gamma^{N}-1} q_{1} u_{2}^{+}-\frac{2\left(\gamma^{N}+1\right)}{\gamma^{N}-1} q_{3} u_{1}^{+} .
\end{aligned}
$$

It follows from (33), (34) that $2\left(u_{1}^{+}\right)^{2}+\left(u_{2}^{+}\right)^{2}=\left(f\left(x_{+}\right)\right)^{2}$, where $f\left(x_{+}\right)$is an arbitrary function and therefore

$$
u_{1}^{+}=f\left(x_{+}\right) \cos \theta, \quad u_{2}^{+}=\sqrt{2} f\left(x_{+}\right) \sin \theta .
$$

Now equations (33), (34) yield to

$$
q_{2}+q_{3}=\frac{1-\gamma^{N}}{\sqrt{2}} \theta_{x_{\gamma}} .
$$

In variables

$$
\theta, \quad u=2 q_{1}, \quad v=-\frac{q_{2}-q_{3}}{\sqrt{2}\left(\gamma^{N}+1\right)}
$$

equations (33)-(37) take the form

$$
\begin{aligned}
& u_{x_{+}}=f\left(x_{+}\right)(\cos \theta)_{x_{\gamma}}, \quad v_{x_{+}}=f\left(x_{+}\right)(\sin \theta)_{x_{\gamma}}, \\
& \theta_{x_{+} x_{\gamma}}=\alpha f\left(x_{+}\right) u \sin \theta+\beta f\left(x_{+}\right) v \cos \theta,
\end{aligned}
$$

where

$$
\alpha=\frac{8 \gamma^{N}}{\left(\gamma^{N}-1\right)^{2}}, \quad \beta=4\left(\frac{\gamma^{N}+1}{\gamma^{N}-1}\right)^{2} .
$$

Changing the variable $x_{+}$and rescaling $x_{\gamma}$ we can set $f\left(x_{+}\right) \rightarrow 1, \alpha \rightarrow \hat{\alpha}=\alpha / \beta$ and $\beta \rightarrow 1$.

The case of the orbit $\mathbb{D}_{N}(0)=\{0, \infty\}$ yields a reducible $L_{0}$ operator

$$
L_{0}=\frac{\mathrm{d}}{\mathrm{d} x_{0}}-p\left(\begin{array}{cc}
0 & \lambda \\
\lambda^{-1} & 0
\end{array}\right), \quad h=\frac{N-1}{2} .
$$

For other values of $h$ the operator is trivial. It is not surprising, since we have imposed the condition that the poles are simple. The result becomes less trivial if we lift this condition (see [17]).

If we take $L_{\gamma}$ and $L_{0}$ as Lax pair, the condition $\left[L_{\gamma}, L_{0}\right]=0$ leads to

$$
p_{x_{\gamma}}=4 q_{1} p, \quad q_{1 x_{0}}=\frac{\gamma^{N}}{2}\left(q_{3}-q_{2}\right), \quad q_{2 x_{0}}=-4 \gamma^{N} q_{1} p, \quad q_{3 x_{0}}=-4 q_{1} p .
$$

In the new variable $u=\log p$, after simple rescaling and a proper coordinates change, the system of equation (39) can be written as

$$
u_{x_{\gamma} x_{0}}=e^{u} \sqrt{1-\left(u_{x_{\gamma}}\right)^{2}} \text {. }
$$

The equation obtained is a well known example of the Liouville type equation [18]. Considering $L_{ \pm}$and $L_{0}$ as Lax pair immediately leads to simple C-integrable system of equations.

For all Lax operators considered in this section the gauge freedom is completely fixed by the reduction group. Only scalar $\lambda$ independent gauge transformations commute with all elements of the reduction group. 
Equations (29) can be transformed into the Sokolov-Golubchik system [19, 20]

$$
\vec{n}_{t}=\vec{n} \times A \vec{m}, \quad \vec{m}_{x}=\vec{m} \times B \vec{n},
$$

where $A$ and $B$ are diagonal matrices, such that $A B=I d$, while equations (38) are a new integrable system of equations, to the best of our knowledge, and may have interesting geometrical applications.

\section{Three examples of $\mathbb{D}_{N}$ reductions, $\mathfrak{g}=\operatorname{sl}(N, \mathbb{C})$}

Let us consider a fairly general Lax pair

$$
\begin{array}{ll}
L(x, t ; \lambda)=\partial_{x}-X(x, t ; \lambda), & X=Q_{0}+Q \lambda+\bar{Q} \lambda^{-1}, \\
M(x, t ; \lambda)=\partial_{t}-T(x, t ; \lambda), & T=P_{0}+P \lambda+\bar{P} \lambda^{-1}+Q^{2} \lambda^{2}+\bar{Q}^{2} \lambda^{-2},
\end{array}
$$

with $Q_{0}, Q, \bar{Q}, P_{0}, P, \bar{P} \in \operatorname{sl}(N, \mathbb{C})$. Note that $Q_{0}$ can always be set to zero by a gauge transformation. In this setting, the compatibility condition (2) yields to the following set of equations:

$$
\begin{array}{ll}
\lambda^{2}: & Q_{x}^{2}=[Q, P] \\
\lambda^{1}: & Q_{t}-P_{x}+\left[Q, P_{0}\right]+\left[\bar{Q}, Q^{2}\right]=0 \\
\lambda^{0}: & P_{0, x}=[Q, \bar{P}]+[\bar{Q}, P] \\
\lambda^{-1}: & \bar{Q}_{t}-\bar{P}_{x}+\left[\bar{Q}, P_{0}\right]+\left[Q, \bar{Q}^{2}\right]=0 \\
\lambda^{-2}: & \bar{Q}_{x}^{2}=[\bar{Q}, \bar{P}] .
\end{array}
$$

The system (43) is a system of $\left(5\left(N^{2}-1\right)\right)$ nonlinear coupled equations for the matrix entries. The group of automorphisms of Lie algebra $\operatorname{sl}(N, \mathbb{C}), N \geqslant 3$ has both inner and outer automorphisms, allowing more possibilities for the realization of the reduction group. Here we consider three different reductions of this system with reduction group isomorphic to $\mathbb{D}_{N}$.

\subsection{Case 1: inner and outer automorphisms}

Let us consider two transformations

$$
s: \quad L(\lambda) \mapsto S^{-1} L(\omega \lambda) S, \quad r: \quad L(\lambda) \mapsto-L^{A}(1 / \lambda),
$$

where $S$ is an $N \times N$ matrix given by $S_{i j}=\delta_{i, j} \omega^{N-i}$, with $\omega=\mathrm{e}^{2 \mathrm{i} \pi / N}$, and where ' $L^{A}$ ' stands for formally adjoint operator $L^{A}=-\partial_{x}+X^{\operatorname{tr}}$. Observe that, neglecting the spectral parameter $\lambda,(44)$ are nothing but two automorphisms of the algebras $\operatorname{sl}(N, \mathbb{C})$; in particular, the first one is an inner automorphism, while the second is outer [15]. They satisfy (18) and therefore generate the dihedral group $\mathbb{D}_{N}$.

Let us now require that both operators $L$ and $M$ are invariant under (44). This leads to algebraic constraints on the matrices $X$ and $T$

$$
\begin{array}{ll}
X(\lambda)=S^{-1} X(\omega \lambda) S, & X(\lambda)=-X^{\operatorname{tr}}(1 / \lambda), \\
T(\lambda)=S^{-1} T(\omega \lambda) S, & T(\lambda)=-T^{\operatorname{tr}}(1 / \lambda),
\end{array}
$$

where 'tr' stands for matrix transposition, which imply

$$
\begin{array}{ll}
P_{0}=0, & \\
\bar{Q}=-Q^{\mathrm{tr}}, & \bar{P}=-P^{\mathrm{tr}}, \\
Q=\mathbf{q}(x, t) \Delta, & P=\mathbf{p}(x, t) \Delta,
\end{array}
$$


where $\mathbf{q}_{i j}=q_{i}(x, t) \delta_{i, j}$ and $\mathbf{p}_{i j}=p_{i}(x, t) \delta_{i, j}$ are diagonal matrices and where $\Delta$ is the shift operator $\Delta_{i j}=\delta_{i, j-1}$. Here and hereafter all indexes are counted modulo $N$. As a consequence, (43) reduces to the following system of $2(N-1)$ nonlinear coupled equations

$$
Q_{t}-P_{x}+\left[\bar{Q}, Q^{2}\right]=0 \quad Q_{x}^{2}=[Q, P]
$$

or, in components,

$$
\begin{aligned}
& q_{i t}-p_{i x}+q_{i} q_{i+1}^{2}-q_{i-1}^{2} q_{i}=0 \\
& q_{i} p_{i+1}-p_{i} q_{i+1}-\left(q_{i} q_{i+1}\right)_{x}=0 .
\end{aligned}
$$

Solutions of (48) can be parametrized by new variables $u_{i}$ and $v_{i}$ (similarly to [8]); indeed, let

$$
q_{i}=\exp \left(u_{i}\right), \quad p_{i}=v_{i} \exp \left(u_{i}\right),
$$

then

$$
v_{i}=-\sum_{r=1}^{N}\left(\frac{2 \bmod (i-r-1, N)+1-N}{2 N}\right)\left(u_{r}+u_{r+1}\right)_{x} .
$$

In the new variables (47) reads

$$
u_{i t}-v_{i x}-u_{i x} v_{i}+\exp \left(2 u_{i+1}\right)-\exp \left(2 u_{i-1}\right)=0, \quad i=1, \ldots, N .
$$

In the case $N=3$ and $Q^{3}=I$ (or $\prod q_{i}=1$ ), equation (46) can be solved explicitly

$$
P=\frac{1}{3}\left[Q, Q Q_{x} Q\right] \text {. }
$$

In components

$$
p_{1}=\frac{1}{3} q_{1}^{2}\left(q_{2} q_{3 x}-q_{2 x} q_{3}\right) \quad p_{2}=\frac{1}{3} q_{2}^{2}\left(q_{3} q_{1 x}-q_{3 x} q_{1}\right) \quad p_{3}=\frac{1}{3} q_{3}^{2}\left(q_{1} q_{2 x}-q_{1 x} q_{2}\right) .
$$

Hence, substituting $p_{i}$ into (47) and rewriting the equations in terms of $u_{i}$ variables we obtain

$$
u_{1 t}=\frac{1}{3}\left(u_{3 x x}-u_{2 x x}\right)+\frac{1}{3} u_{1 x}\left(u_{3 x}-u_{2 x}\right)-\exp \left(2 u_{2}\right)+\exp \left(2 u_{3}\right)
$$

and cyclic permutations of the indexes $1,2,3$.

\subsection{Case 2: inner automorphisms}

Let us now turn our attention to a different symmetry conditions

$$
L(\lambda)=S^{-1} L(\omega \lambda) S, \quad L(\lambda)=R^{-1} L(1 / \lambda) R,
$$

where $R_{i j}=\delta_{i, N-j}$ (all indexes are counted modulo $N$ ). Conditions (54) are simultaneous inner automorphisms. In this case

$$
\begin{array}{lc}
\left(P_{0}\right)_{i j}=p_{0 i} \delta_{i, j}, & \\
\bar{Q}=R Q R, & \bar{P}=R P R, \\
Q=\mathbf{q}(x, t) \Delta, & P=\mathbf{p}(x, t) \Delta .
\end{array}
$$

Hence (43) reduces to

$$
\begin{aligned}
& Q_{t}-P_{x}+\left[Q, P_{0}\right]+\left[\bar{Q}, Q^{2}\right]=0 \\
& P_{0 x}=[Q, \bar{P}]+[\bar{Q}, P] \\
& Q_{x}^{2}=[Q, P]
\end{aligned}
$$


or, in components

$$
\begin{aligned}
& q_{i t}=p_{i x}+p_{0 i} q_{i}-q_{i} p_{0 i+1}+q_{i} q_{i+1} q_{N-i-2}-q_{N-i} q_{i-1} q_{i} \\
& p_{0 i x}=q_{i} p_{N-i-1}-p_{i} q_{N-i-1}+q_{N-i} p_{i-1}-p_{N-i} q_{i-1} \\
& q_{i} p_{i+1}-p_{i} q_{i+1}-\left(q_{i} q_{i+1}\right)_{x}=0 \quad i=1, \ldots, N .
\end{aligned}
$$

For $N=3$ we can use the result (52) for $P$ to solve (56) and find

$$
P_{0}(x, t)=q_{2}(x, t) q_{3}(x, t) \operatorname{diag}\{1,1,-2\} .
$$

In $u_{i}$ variables (49), we obtain

$$
\begin{aligned}
& u_{1 t}=\frac{1}{3}\left(u_{3 x x}-u_{2 x x}\right)+\frac{1}{3} u_{1 x}\left(u_{3 x}-u_{2 x}\right) \\
& u_{2 t}=\frac{1}{3}\left(u_{1 x x}-u_{3 x x}\right)+\frac{1}{3} u_{2 x}\left(u_{1 x}-u_{3 x}\right)-\exp \left(2 u_{1}\right)+4 \exp \left(u_{3}+u_{2}\right) \\
& u_{3 t}=\frac{1}{3}\left(u_{2 x x}-u_{1 x x}\right)+\frac{1}{3} u_{3 x}\left(u_{2 x}-u_{1 x}\right)-4 \exp \left(u_{3}+u_{2}\right)+\exp \left(2 u_{1}\right) .
\end{aligned}
$$

\subsection{Case 3: symplectic automorphisms}

In even dimensions $N=2 n$ we can consider a third case given by

$$
L(\lambda)=S^{-1} L(\omega \lambda) S \quad L(\lambda)=-J^{-1} L^{A}(1 / \lambda) J
$$

where $J$ is the symplectic matrix $J=\left(\begin{array}{cc}0 & I \\ -I & 0\end{array}\right)$. From (63) it follows that $P_{0}$ is a diagonal matrix

$$
P_{0}=\operatorname{diag}\left\{p_{01}(x, t), \ldots, p_{0 n}(x, t),-p_{01}(x, t), \ldots,-p_{0 n}(x, t)\right\}
$$

while

$$
Q=\left(\begin{array}{ccccc}
0 & q_{1} & 0 & \ldots & 0 \\
0 & 0 & q_{2} & \ldots & 0 \\
0 & 0 & 0 & \ddots & 0 \\
0 & 0 & 0 & \ddots & q_{2 n-1} \\
-q_{2 n} & 0 & \ldots & 0 & 0
\end{array}\right) \quad P=\left(\begin{array}{ccccc}
0 & p_{1} & 0 & \ldots & 0 \\
0 & 0 & p_{2} & \ldots & 0 \\
0 & 0 & 0 & \ddots & 0 \\
0 & 0 & 0 & \ddots & p_{2 n-1} \\
-p_{2 n} & 0 & \ldots & 0 & 0
\end{array}\right)
$$

and

$$
\bar{Q}=-J^{-1} Q^{\mathrm{tr}} J \quad \bar{P}=-J^{-1} P^{\operatorname{tr}} J .
$$

The equations for this case read

$$
\begin{aligned}
& q_{i t}=p_{i x}+q_{i} p_{0 i}-p_{0 i+1} q_{i}+q_{n+i-1} q_{i-1} q_{i}-q_{i} q_{i+1} q_{n+i+1} \\
& p_{0 i x}=-q_{i} p_{n+i}+q_{n+i} p_{i}+q_{i-1} p_{n+i-1}-q_{n+i-1} p_{i-1} \\
& q_{i} p_{i+1}-p_{i} q_{i+1}-\left(q_{i} q_{i+1}\right)_{x}=0, \quad i=1, \ldots, 2 n .
\end{aligned}
$$

For $n=2$ we have $\left[\bar{Q}, Q^{2}\right]=0$ and equation (65) simplifies. In terms of $u_{i}$ variables (49) and (50) the system becomes

$$
\begin{aligned}
& w_{1 t}+\frac{1}{2}\left(w_{2 x} w_{3 x}\right)=0 \\
& w_{2 t}+w_{3 x x}+\frac{1}{2}\left(w_{3 x} w_{1 x}\right)-2 l_{2}=0 \\
& w_{3 t}-w_{2 x x}+\frac{1}{2}\left(w_{2 x} w_{1 x}\right)-2 l_{1}=0 \\
& l_{1 x}=2 w_{2 x} \exp \left(-w_{1}\right), \quad l_{2 x}=-2 w_{3 x} \exp \left(w_{1}\right),
\end{aligned}
$$

where $w_{1}=u_{1}+u_{3}, w_{2}=u_{1}-u_{3}, w_{3}=u_{2}-u_{4}$ and where $l_{1}=\left(p_{01}+p_{02}\right), l_{2}=\left(p_{01}-p_{02}\right)$.

System (68) is new, to the best of our knowledge. Moreover, for $N$ general all systems of equations obtained in this sections can be regarded as new examples of lattice equations. 


\section{Conclusions and remarks}

All properties of integrable equations are encoded in their Lax representations. Therefore, the description of the variety of integrable equations and their reductions can be pursued starting from the theory of Lax operators. In particular, the problem of reductions can be studied imposing symmetry conditions on the Lax operators, reducing the corresponding nonlinear systems of equations to smaller subsystems. Symmetries of Lax representations form a group - the reduction group [8-10, 17]. The reduction group approach has been used to describe Lie-algebraic reductions of the $N$-waves equation [11], to find Lax representation for a number of new nonlinear Schrödinger type equations [21] and to build explicit solutions for the Landau-Lifschits equation [22]. In this paper we have revisited the reduction group approach, illustrated it by a number of examples and motivated our further study. There are several natural directions for development.

The equations obtained in the previous section are integrable for any $N$. Assuming $N \rightarrow \infty$ and taking continuous limits one can find $(2+1)$ dimensional integrable equations (and corresponding Lax representations - all structures, such as symmetries, conservation laws, etc can be recomputed in these limits). There are several ways to take a continuous limit, the result depends on the balance of nonlinearity and dispersion. For example, the KadomtsevPetviashvili equation

$$
u_{T}=\frac{1}{3} u_{y y y}-D_{y}^{-1}\left(u_{X X}\right)+6 u u_{y}
$$

can be recovered from (47), (48) as a continuous limit if we assume $q=\exp \left(h^{2} u\right)$, where $h$ is the lattice step, and perform a Galilean transformation. On the other side, performing rather naive expansions $q_{i \pm 1}=q \pm h q_{y}+O\left(h^{2}\right)$ and $p_{i \pm 1}=p \pm h p_{y}+O\left(h^{2}\right)$, we would receive, after proper rescaling, the hydrodynamic type equation

$$
u_{t}=D_{y}\left\{\left(D_{y}^{-1} D_{x}\right)^{2} u+\frac{1}{2}\left(D_{y}^{-1} D_{x} u\right)^{2}+\mathrm{e}^{(2 u)}\right\} .
$$

Similarly, equations (58)-(60) yield to the following systems of equations in $(2+1)$ dimensions:

$u_{t}=D_{y}\left\{\left(D_{y}^{-1} D_{x}\right)^{2} u+\frac{1}{2}\left(D_{y}^{-1} D_{x} u\right)^{2}+D_{x}^{-1} D_{y}\left[\mathrm{e}^{(u+v)} D_{y}^{-1} D_{x}(u+v)\right]+\mathrm{e}^{(u+v)}\right\}$
$v_{t}=-D_{y}\left\{\left(D_{y}^{-1} D_{x}\right)^{2} v+\frac{1}{2}\left(D_{y}^{-1} D_{x} v\right)^{2}+D_{x}^{-1} D_{y}\left[\mathrm{e}^{(u+v)} D_{y}^{-1} D_{x}(u+v)\right]+\mathrm{e}^{(u+v)}\right\}$

and (65)-(67) yield to

$u_{t}=D_{y}\left\{\left(D_{y}^{-1} D_{x}\right)^{2} u+\frac{1}{2}\left(D_{y}^{-1} D_{x} u\right)^{2}+D_{x}^{-1} D_{y}\left[\mathrm{e}^{(u+v)} D_{y}^{-1} D_{x}(u-v)\right]+\mathrm{e}^{(u+v)}\right\}$

$v_{t}=D_{y}\left\{\left(D_{y}^{-1} D_{x}\right)^{2} v+\frac{1}{2}\left(D_{y}^{-1} D_{x} v\right)^{2}+D_{x}^{-1} D_{y}\left[\mathrm{e}^{(u+v)} D_{y}^{-1} D_{x}(u-v)\right]+\mathrm{e}^{(u+v)}\right\}$.

Note that (71) can be obtained from (73) by setting $v=u$.

A simple modification of the procedure enables us to study Lax operators with noncommutative (matrix) entries. For example, we can treat elements of $\operatorname{sl}(2 M, \mathbb{C})$, as $2 \times 2$ matrices with $M \times M$ matrix entries. The corresponding reduced systems can be viewed as a system of equations for non-Abelian variables. The following system of equations

$$
\begin{aligned}
& \mathbf{q}_{0 t}-\mathbf{p}_{0 x}+\left[\mathbf{q}_{0}, \mathbf{p}_{0}\right]+\left[\mathbf{q}_{1}, \mathbf{p}_{1}\right]=0 \\
& \mathbf{q}_{1 t}+\left[\mathbf{q}_{1}, \mathbf{p}_{0}\right]+\left\{\mathbf{q}_{2}, \mathbf{p}_{2}\right\} / 4=0 \\
& \mathbf{q}_{2 t}+\left[\mathbf{q}_{2}, \mathbf{p}_{0}\right]+\left\{\mathbf{q}_{1}, \mathbf{p}_{2}\right\}=0 \\
& \mathbf{p}_{1 x}+\left[\mathbf{p}_{1}, \mathbf{q}_{0}\right]+\left\{\mathbf{q}_{2}, \mathbf{p}_{2}\right\} / 4=0 \\
& \mathbf{p}_{2 x}+\left[\mathbf{p}_{2}, \mathbf{q}_{0}\right]+\left\{\mathbf{q}_{2}, \mathbf{p}_{1}\right\}=0,
\end{aligned}
$$


where the variables $\mathbf{q}_{i}, \mathbf{p}_{i}$ are elements of a non-commutative free algebra (or matrices of any size) and $\left\{\mathbf{q}_{i}, \mathbf{p}_{j}\right\}=\mathbf{q}_{i} \mathbf{p}_{j}+\mathbf{p}_{j} \mathbf{q}_{i}$ is a non-Abelian generalization of (31) (the Bäcklund transformations for the sinh-Gordon equation). In the non-Abelian case we have to fix the gauge freedom further in order to make equations (74) well determined.

A very important issue is the solution of nonlinear integrable models. The reduction group proves to be not only a very useful tool to find new integrable equations and classify Lax pairs but also a necessary instrument in this context. Indeed, without the constraints imposed by the reduction group on the spectral data it is not even possible to formulate the inverse problem, which would lead to explicit solutions of the integrable equation. The general setup of the correspondence between reduction groups and analyticity properties of the spectral data is one of the next issues on our research agenda.

Another challenging problem is the study of automorphic Lie algebras in a pure algebraic way. They can be always linked back to Lax operators, Baxter's $R$-matrix equations, etc. Moreover, the problem of a complete description of rational automorphic Lie algebras seems to be feasible. Our optimizm is based upon a simple group-theoretical observation [17] and a remarkable theorem of Felix Klein [23]: the complete list of finite groups of fractional-linear transformations is given by the cyclic group $\mathbb{Z}_{N}$, the dihedral group $\mathbb{D}_{N}$ and the groups of symmetry of Plato solids, i.e. the tetrahedral group, the octahedral group and the icosahedral group. It would be interesting to generalize the theory of automorphic Lie algebras to the cases of elliptic and higher genus algebraic curves. We believe that automorphic Lie algebras will find applications far beyond the theory of Lax operators.

\section{Acknowledgments}

We would like to thank the Newton Institute for Mathematical Sciences where we started to work on this paper. The initial stage of the work of S L was supported by the University of Leeds William Wright Smith scholarship and successively by a grant of the Swedish foundation Blanceflor Boncompagni-Ludovisi, née Bildt, for which S L is most grateful. The work of A M was partially supported by the RFBR grant 02-01-00431. Both authors thank V V Sokolov for discussions and useful comments.

\section{References}

[1] Manakov S V, Novikov S P, Pitajevski L P and Zakharov V E 1980 Theory of Soliton. The Inverse Problem Method (Moskov: Nauka) (Russian)

Manakov S V, Novikov S P, Pitajevski L P and Zakharov V E 1984 Theory of Solution. The Inverse Problem Method (New York: Plenum) (Engl. Transl.)

[2] Ablowitz M J and Segur H 1981 Solitons and the Inverse Scattering Transform (SIAM-Studies in Applied Mathematics) (Philadelphia: SIAM) p 189

[3] Calogero F and Degasperis A 1982 Spectral Transform and Solitons Vol 1 (Amsterdam: North-Holland)

[4] Wahlquist H D and Estabrook F B 1973 Bäcklund transformation for solution of the Korteweg-de Vries equation Phys. Rev. Lett. 23 1386-9

[5] Wahlquist H D and Estabrook F B 1975 Prolongation structures and nonlinear evolution equations J. Math. Phys. 16 1-7

[6] Wahlquist H D and Estabrook F B 1976 Prolongation structures and nonlinear evolution equations J. Math. Phys. 17 1293-7

[7] Flaschka H, Newell A C and Tabor M 1991 Integrability What is Integrability? (Springer Series in Nonlinear Dynamics) ed V E Zakharov (Berlin: Springer) pp 73-114

[8] Mikhailov A V 1979 On the integrability of two-dimensional generalisation of the Toda Lattice Lett. J. Exp. Theor. Phys. 30 443-8

[9] Mikhailov A V 1981 The reduction problem and the inverse scattering method Soliton Theory Proc. SovietAmerican Symp. Soliton Theory (Kiev USSR 1979) Physica D 1-2 73-117 
[10] Mikhailov A V 1980 Reduction in Integrable Systems. The Reduction Group Pisma ZETP 32 187-92

[11] Gerdjikov V S, Grahovski G G and Kostov A N 2001 Reductions of N-waves interaction related to low rank simple Lie algebras $Z_{2}$-Reductions J. Phys. A: Math. Gen. 34 9425-61

[12] Mikhailov A V and Zakharov V E 1978 Relativistically invariant models of field theory, integrable by inverse transform method J. Exp. Theor. Phys. 74 1953-73

[13] Shabat A B and Zakharov V E 1979 Integration of nonlinear equations of mathematical physics by the method of the inverse scattering problem. II Funct. Anal. Appl. 13 166-74

[14] Takhtajan L A and Zakharov V E 1979 Equivalence of the nonlinear Schrodinger equation and the Heisenbergferromagnet equation Theor. Math. Phys. 38 17-23

[15] Jacobson N 1961 Lie Algebras Interscience (New York: Wiley)

[16] Zakharov V E and Mikhailov A V 1983 Inverse scattering method with spectral parameter on algebraic curves Funct. Anal. Appl. 17 1-6

[17] Lombardo S and Mikhailov A V Reduction groups and automorphic Lie algebras (in preparation)

[18] Zhiber A V and Sokolov V V 2001 Exactly integrable hyperbolic equations of Liouville type Russian Math. Surveys 56 61-101

[19] Sokolov V V and Golubchik I Z 2000 Yet another kind of classical Yang-Baxter Equation Funkts. Anal. Prilozhen. 34 75-8

[20] Sokolov V V and Golubchik I Z 2002 Compatible Lie Brackets and integrable equations of the principal Chiral Model type Funct. Anal. Appl. 36 172-81

[21] Mikhailov A V, Shabat A B and Yamilov R I 1987 The symmetry approach to the classification of non-linear equations. Complete lists of integrable systems Russian Math. Surveys 42 1-63

[22] Mikhailov A V 1982 The Landau-Lifschitz equation and the riemann boundary problem on a torus Phys. Lett. A 9251

[23] Klein F 1875 Über binäre Formen mit linearen Transformationen sich selbst Mathematische Annalen Bd 9 